\title{
Mandibular Fracture in Conjunction with Bicortical Penetration, Using Wide-Diameter Endosseous Dental Implants
}

\author{
Won-suk Oh, DDS, MS, ${ }^{1}$ Eleni D. Roumanas, DDS, ${ }^{2}$ \& John Beumer III, DDS, $\mathrm{MS}^{2}$ \\ ${ }^{1}$ Division of Prosthodontics, Department of Biologic and Materials Sciences, University of Michigan School of Dentistry, Ann Arbor, MI \\ ${ }^{2}$ Division of Advanced Prosthodontics, Biomaterials and Hospital Dentistry, UCLA School of Dentistry, Los Angeles, CA
}

\begin{abstract}
Keywords
Prosthodontic rehabilitation; fracture of atrophic edentulous mandible; thermal injury; wide-diameter implant; bicortical penetration.

\section{Correspondence \\ Won-suk Oh, Department of Biologic \& Materials Sciences, University of Michigan School of Dentistry, 1011 N. University, Rm K1014A, Ann Arbor, MI 48109-1078. E-mail: ohws@umich.edu}

This clinical report was presented at the biennial session of the ICP in Fukuoka, Japan, September 2007.

Accepted December 2, 2009

\begin{abstract}
Prosthodontic rehabilitation of a patient with an atrophic edentulous mandible presents a significant challenge in restoring esthetics and function. The purpose of this clinical report is to describe fracture of an atrophic edentulous mandible opposing maxillary natural dentition in association with endosseous dental implants. The patient received two wide-diameter implants in the anterior mandible for an implant-assisted mandibular overdenture, in which the implants penetrated the inferior border of the mandible for bicortical stabilization. Three months following implant placement surgery, the patient experienced pain, swelling, and intraoral purulent drainage around the right implant. Panoramic radiograph revealed a fracture of the mandible through the right implant site and signs of infection around the left implant. The implants were removed surgically, and open reduction and fixation of the fracture site were undertaken using a titanium bone fixation plate. This clinical report demonstrates that placement of widediameter implants in conjunction with bicortical penetration in a severely atrophic edentulous mandible can risk fracture of the mandible.
\end{abstract}

doi: 10.1111/j.1532-849X.2010.00646.x

Osseointegrated dental implants are highly predictable for prosthetic rehabilitation of the edentulous mandible, ${ }^{1-5}$ although various biologic and mechanical complications have been reported. ${ }^{6-9}$ Mandibular fracture is not a common problem encountered in conjunction with endosseous implants but recognized as a serious potential complication. This is particularly problematic in the highly resorbed, osteoporotic, or osteosclerotic edentulous mandible. ${ }^{10-13}$

In the normal human dentate mandible, the volume of cortical bone is approximately 3.5 times less than that of cancellous bone. ${ }^{14}$ Following the loss of teeth, the mandible experiences significant reduction in bone height and volume. Although both cortical and cancellous portions of the mandible are affected by resorption, the remaining portion of the atrophic mandible becomes denser with increased load applications from the mandibular prosthesis. The resulting increase in cortical wall thickness and bone density occurs simultaneously with vertical bone resorption, beneath the functioning prosthesis. ${ }^{15,16}$ The atrophic dense edentulous mandible subsequently becomes susceptible to fracture from external trauma or functional forces.

During placement of endosseous implants, gentle surgical technique and adequate irrigation and blood supply are prerequisite to the process of osseointegration. ${ }^{17-19}$ The traditional healing period for this process is approximately 4 months for machined surfaces, but currently available moderately rough surfaces may accelerate bone formation and enhance biomechanical and biochemical bonding around the implant. Prior to establishment of osseointegration, the implants are required to maintain intimate contact with the bone by means of frictional fit. Thus, stress distribution is not homogenous at the implant sites, which act as stress concentrators, in particular in the atrophic edentulous mandible due to reduced bone volume. ${ }^{20-23}$ Fracture of the atrophic mandible can occur even under normal function, in part due to flexure of the mandible by physiologic forces generated by the muscles of mastication. ${ }^{24}$

Bicortical engagement is recommended and frequently used in the atrophic edentulous mandible to enhance implant stability, permit placement of longer implants, and improve load transfer characteristics, ${ }^{25-27}$ however, bicortical penetration of the atrophic mandible may significantly reduce structural integrity due to the loss of continuity at the inferior and lateral border. ${ }^{28,29}$ Implant site preparation for wide-diameter implants may further increase the risk of thermal injury of the bone and thin out the buccal and lingual cortices creating an "egg crate" configuration. ${ }^{30}$ The purpose of this clinical report is to describe the fracture of a patient's atrophic edentulous mandible after placement of two wide-diameter implants 
that penetrated the inferior border of the mandible and that opposed a natural maxillary dentition under normal function. This report also reviewed preoperative strategies for prosthodontic rehabilitation.

\section{Clinical report}

The patient, a 63-year-old white man, presented to the Maxillofacial Prosthetics Clinic at the University of California, Los Angeles in July 2004, complaining of difficulty wearing his conventional mandibular complete denture (CD) and desiring dental implant-assisted overdenture (IOD). Past medical history included mild chronic obstructive pulmonary disease, heart murmur, and hypertension. The patient had suffered a gunshot wound in the mandible with the entry wound on the left and exit wound on the right, approximately 30 years earlier, for which he underwent multiple reconstructive surgeries, including a bone graft with a titanium mesh in the right posterior mandible. The last mandibular surgery was in 1990 to resolve the infection caused by oral exposure of the retained Ti mesh used for grafting.

Intraoral examination revealed natural dentition in the maxilla and a severely atrophic edentulous mandible (Prosthodontic Diagnostic Index, Edentulous Class IV). The preexisting conventional mandibular CD was neither stable nor retentive. Cephalometric and panoramic radiographic examination revealed advanced resorption of the mandible with deficient bone ( $7 \mathrm{~mm}$ in height) in the symphyseal area (Figs 1 and 2). The patient was informed of the possible consequences of prosthodontic rehabilitation of the edentulous mandible without addressing the maxillary dentition. These included difficulty in establishing proper occlusion, compromised stability of the mandibular denture, and increased bone resorption of the mandible. The proposed treatment options were as follows: conventional maxillary/mandibular CDs; maxillary CD opposing mandibular 2 IOD (2-IOD), 4 IOD (4-IOD), or fixed bone anchored bridge; maxillary/mandibular 2-IOD or 4-IOD; or do nothing. Onlay grafting procedures to augment the mandible were ruled out because of anticipated accelerated bone graft resorption and the patient's past surgical history. Due to financial limi-

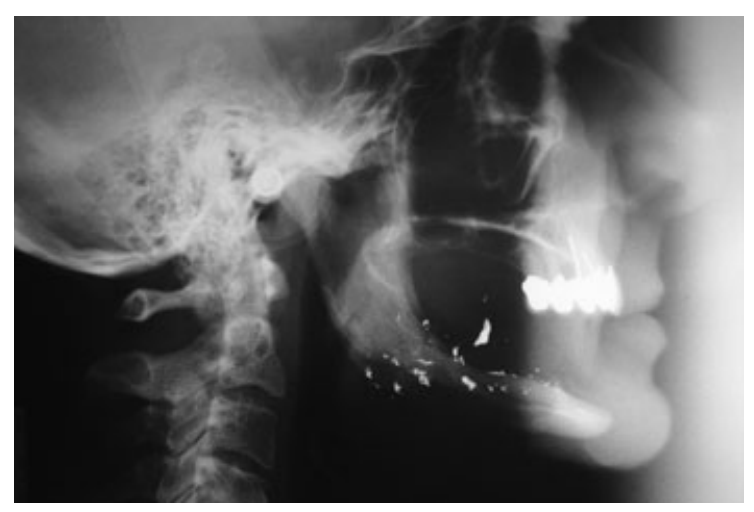

Figure 1 Preoperative cephalometric radiographic image exhibiting natural dentition in maxilla, atrophic edentulous mandible, and opaque particles from gunshot wound in posterior mandible.

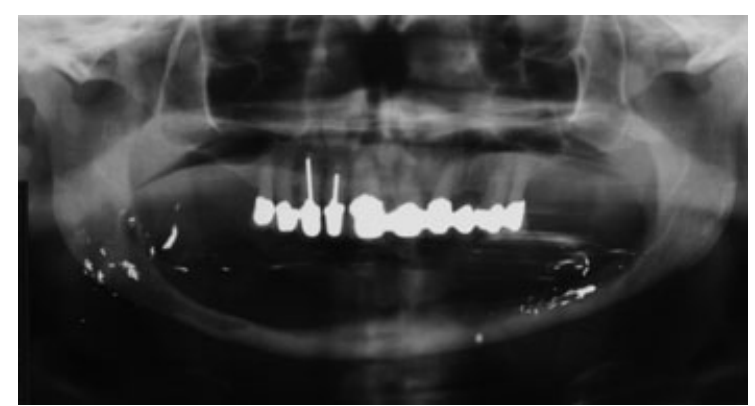

Figure 2 Preoperative panoramic radiographic image exhibiting advanced atrophy of the mandible ( $7 \mathrm{~mm}$ height of anterior mandible).

tations the patient chose the mandibular 2-IOD, retained with a tissue bar for the edentulous mandible. He desired to maintain his natural dentition in the maxilla. The patient's existing mandibular CD was relined using a tissue conditioner (Viscogel, Dentsply DeTrey GmbH, Konstanz, Germany) to provide stability and restore occlusal vertical dimension. The prosthesis was tested in the mouth for esthetics and function and duplicated in a clear acrylic resin (Teets, Co-Oral-Ite Dental Mfg Co, Diamond Springs, CA) using a denture duplicator flask kit (Lang Dental Mfg, Wheeling, IL). The duplicate denture was modified to a surgical template and used during the surgical procedure to direct implant position.

Wide-diameter implants (5.0-mm diameter) were selected to increase the contact surface area of the implant with the bone. Under local anesthesia, a crestal incision was made and a mucoperiosteal flap was reflected between the mental foramen. Gentle surgical technique was used to create implant osteotomy sites under copious irrigation during drilling. Two short (8.5-mm length) two-stage endosseous screw-type implants (Osseotite, 3i, Palm Beach Gardens, FL) were placed in the right and left mandibular canine sites where the implants engaged and penetrated the inferior border of the mandible (Fig 3). The implants were submerged and allowed to heal undisturbed. The patient did not wear his denture for 1 week postoperatively. Following this period, the intaglio surface of the existing denture was aggressively relieved and relined with a resilient tissue conditioning material (Visco-gel), which was replaced every other week. The patient was instructed to maintain a soft

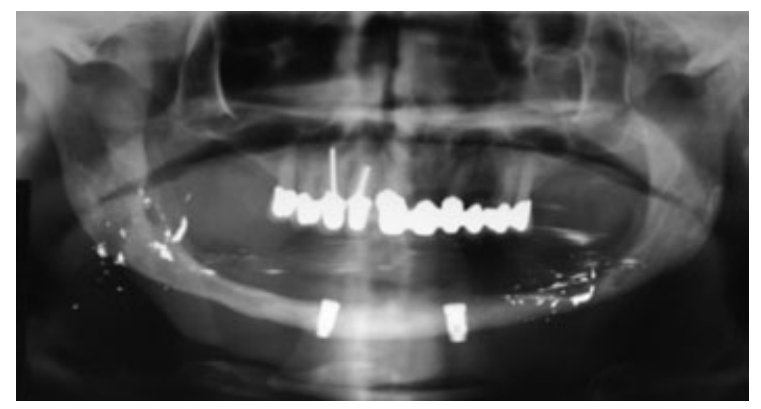

Figure 3 Postoperative, panoramic radiographic image exhibiting two screw-type endosseous wide-diameter implants in each canine area, penetrating inferior border of mandible. 


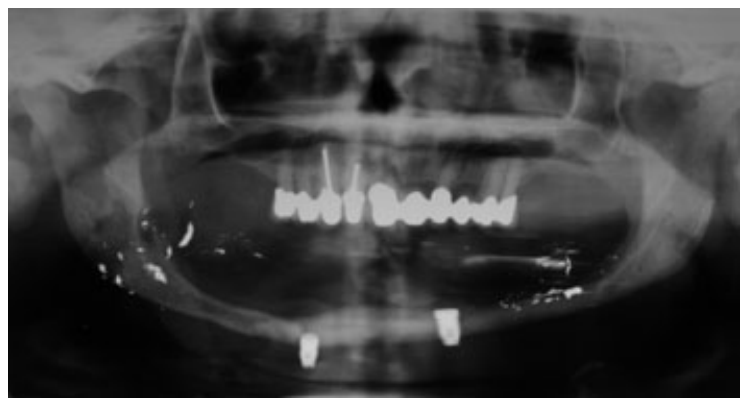

Figure 4 Panoramic radiographic image exhibiting displacement of right implant, along with fracture of mandible at implant site and radiolucency around left implant.

diet and remove the denture from his mouth at night. Over the next months, in the right symphyseal area, the patient experienced several episodes of inflammation and associated pain, which appeared to subside with antibiotics.

Three months following surgery, the patient presented with pain and swelling in the right submandibular area. Intraoral examination revealed hyperplastic granulation tissue and purulent exudate from the periimplant area in the right mandible. A panoramic radiograph showed evidence of a right mandibular fracture, displacement of the right implant into the submandibular soft tissue space, and signs of infection around the left implant (Fig 4). Review of the medical history did not reveal any contributory findings, and the patient did not report a history of external trauma to the region. The patient was placed on Clindamycin (Cleocin, Pfizer Inc, New York, NY), 300 mg orally, four times daily for 7 days and advised to remain on a pureed, soft diet.

An open reduction was planned to remove both implants and reduce the mandibular fracture. Under local anesthesia and IV sedation, the left implant was identified and removed without complications. The right implant site was noted to have a mandibular fracture with the implant displaced in the submandibular area. The implant was removed, and the fracture site was debrided of all reactive granulation tissue. The bony fragments were approximated and stabilized with a $1.7-\mathrm{mm}$ 4-hole Ti bone fixation plate (Arthrex Inc, Naples, FL) and 6-mm bone screws (Arthrex Inc). A 2.0-mm-wide and 12-mm-

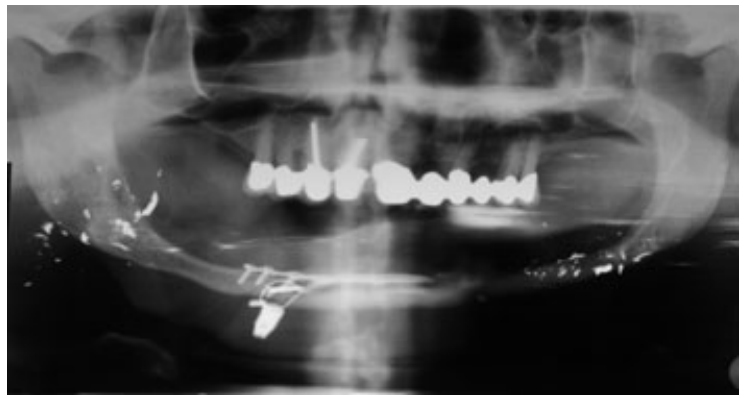

Figure 5 Panoramic radiographic image exhibiting fixation of mandible with titanium plate and displaced right implant in submandibular soft tissue.

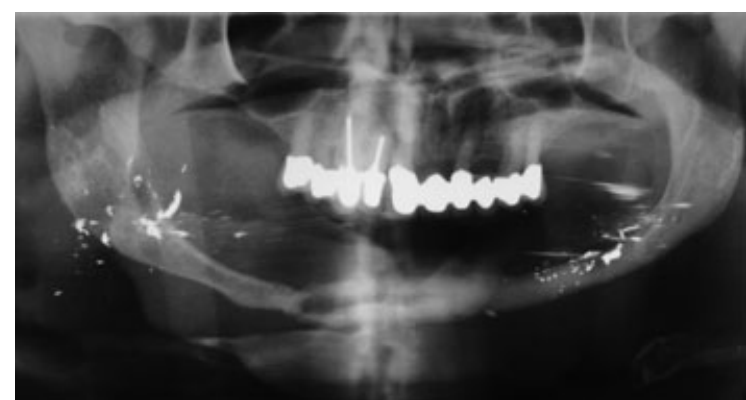

Figure 6 Panoramic radiographic image exhibiting reunion of fractured mandible and removal of hardware.

long lag screw (Arthrex Inc) was also placed (Fig 5). Following reduction, the fracture was noted to have good stability, the region was irrigated copiously, and the intraoral incision was closed in a watertight manner. The patient was instructed not to use the denture for 6 weeks and placed on Clindamycin, $300 \mathrm{mg}$ four times daily for 7 days.

The patient's postoperative course was uneventful, and he was discharged from the hospital two days later. He was recalled biweekly, until an improvement in symptoms was noted with no evidence of extraoral swelling or lymphadenopathy. At the 6-week follow-up appointment, a follow-up panoramic radiograph was made, showing evidence of good bony alignment, bony callus formation, and healing around the fracture site. The patient did not report discomfort or other adverse symptoms. The existing denture was relined with a tissue conditioner (Visco-gel), and the patient continued on a soft diet regimen. Approximately 4 months later, the hardware was removed, and following complete healing of the soft tissues, a new mandibular conventional CD was fabricated (Fig 6).

\section{Discussion}

Implant site preparation and placement should be gentle and atraumatic with copious irrigation for successful osseointegration, particularly in the anterior portion of the atrophic edentulous mandible with dense cortical bone. ${ }^{2,3}$ Inadequate irrigation and cooling while drilling in dense cortical bone may result in elevated bone temperatures without apparent visual evidence, leading to bone necrosis with compromised and delayed healing at the implant-bone interface. ${ }^{17,19}$ The risk of thermal injury and resulting lack of osseointegration can be greater in site preparations for wide-diameter implants with their increased drill size and peripheral speeds, especially in dense cortical bone with reduced vascularity. ${ }^{2,3,17,19}$ Thus, operator experience and judgment are critical in determining bone density. Using sharp drills of incremental size, gentle surgical technique, and light and intermittent drilling pressure with copious irrigation are critical to avoid the harmful effects of temperature increase. ${ }^{17,19,31}$

Wide-diameter implant site preparation and placement in the atrophic mandible may alter the critical amount of remaining bone needed to maintain mandibular integrity and prevent fracture with loading, yet it could predispose the jaw to breakage, especially if the bone were subject to overheating and uneven stress distribution. ${ }^{10-13,21-23}$ The stress 
distribution pattern may be unfavorable around the implant during the healing period, until hard and dense lamellar bone replaces soft woven bone. ${ }^{21-23}$ The combination of widediameter osteotomies resulting in inadequate remaining bone volume of the atrophic mandible, coupled with bone overheating could have contributed to the lack of implant integration and mandibular fracture. The implants, although not immediately loaded, were subjected to functional loading from the denture soft liner through the soft tissue, by the opposing natural dentition.

Wide-diameter implants are often chosen to provide prosthesis support when vertical height of bone is not available, but bone volume is present. ${ }^{25,29,30}$ Short wide-diameter implants appear to withstand functional loading and maintain stable marginal bone level, but may require a longer healing period before supporting the prosthesis. ${ }^{3,4,8}$ The load bearing requirements of implants assisting an IOD are reduced as forces of occlusion are shared between the implants and the mucoperiosteum. Implant survival for short implants was significantly greater than for longer implants placed in augmented mandible with an autologous bone graft. ${ }^{3,8}$ The lack of cancellous bone and blood supply in the anterior mandible led to necrosis of the poorly adapted graft and loss of the implants during the healing phase. ${ }^{3}$ Distraction osteogenesis is an alternative mode of treatment in increasing vertical height of an atrophic edentulous mandible; however, care must be taken to minimize potential complications such as fracture of the mandible, neurological disturbances, and resorption of the transport segment. ${ }^{9}$

Increasing the number of implants does not appear critical in reducing the stress in an atrophic mandible, nor were significant differences noted in stress distribution in the bone containing two or four implants. ${ }^{20}$ When two implants are placed bilaterally, the canine sites are usually chosen for implant placement depending on the arch size, shape, and jaw relationship, and for additional implants if placed at a later date. ${ }^{4,7,20}$ Reported prosthetic complications were minor for tissue-bar retained IODs in the atrophic edentulous mandible, ${ }^{4}$ and the retention and stability of the IOD did not diminish significantly over time. ${ }^{7}$ In general, restoration of an atrophic edentulous mandible with 2-IOD appears to increase patient's expectations and reduce direct and aftercare costs, although the long-term effect of posterior mandibular resorption by means of the IOD is yet to be determined. ${ }^{1,5}$

\section{Conclusion}

Placement of wide-diameter implants in conjunction with bicortical penetration may jeopardize the structural integrity of the severely atrophic mandible; therefore, computed tomography (CT) planning is important for proper implant placement planning. Inadequate remaining bone volume after placement of wide-diameter implants increases stress concentration from functional loading, or thermal bone injury from implant surgery may contribute to mandible fracture at the implant site. Implants may engage the inferior portion of the mandible for stability, but the bone volume and density when penetrating the inferior border of a severely atrophic mandible should be considered. Careful case selection, the use of CT planning, and patient awareness of potential complications are necessary prerequisites when contemplating this procedure.

\section{References}

1. Winkler S, Monasky GE: The edentulous mandible opposing maxillary natural teeth: treatment considerations utilizing implant overdentures. Implant Dent 1993;2:44-47

2. Keller EE: Reconstruction of the severely atrophic edentulous mandible with endosseous implants: a 10-year longitudinal study. J Oral Maxillofac Surg 1995;53:305-320

3. Stellingsma C, Meijer HJ, Raghoebar GM: Use of short endosseous implants and an overdenture in the extremely resorbed mandible: a five-year retrospective study. J Oral Maxillofac Surg 2000;58:382-387

4. Stellingsma K, Raghoebar GM, Meijer HJ, et al: The extremely resorbed mandible: a comparative prospective study of 2-year results with 3 treatment strategies. Int J Oral Maxillofac Implants 2004; 19:563-577

5. Stoker GT, Wismeijer D, van Waas MA: An eight-year follow-up to a randomized clinical trial of aftercare and cost-analysis with three types of mandibular implant-retained overdentures. J Dent Res 2007;86:276-280

6. Goodacre CJ, Kan JY, Rungcharassaeng K: Clinical complications of osseointegrated implants. J Prosthet Dent 1999;81:537-552

7. Timmerman R, Stoker GT, Wismeijer D, et al: An eight-year follow-up to a randomized clinical trial of participant satisfaction with three types of mandibular implant-retained overdentures. J Dent Res 2004;83:630-633

8. Tawil G, Aboujaoude N, Younan R: Influence of prosthetic parameters on the survival and complication rates of short implants. Int J Oral Maxillofac Implants 2006;21:275-282

9. Raghoebar GM, Stellingsma K, Meijer HJ, et al: Vertical distraction of the severely resorbed edentulous mandible: an assessment of treatment outcome. Int J Oral Maxillofac Implants 2008;23:299-307

10. Mason ME, Triplett RG, Van Sickels JE, et al: Mandibular fractures through endosseous cylinder implants: report of cases and review. J Oral Maxillofac Surg 1990;48:311-317

11. Tolman DE, Keller EE: Management of mandibular fractures in patients with endosseous implants. Int J Oral Maxillofac Implants 1991;6:427-436

12. Shonberg DC, Stith HD, Jameson LM, et al: Mandibular fracture through an endosseous implant. Int J Oral Maxillofac Implants 1992;7:401-404

13. Thaller SR: Fractures of the edentulous mandible: a retrospective review. J Craniofac Surg 1993;4:91-94

14. Iwashita Y: Basic study of the measurement of bone mineral content of cortical and cancellous bone of the mandible by computed tomography. Dentomaxillofac Radiol 2000;29:209-215

15. Solar P, Ulm CW, Thornton B, et al: Sex-related differences in the bone mineral density of atrophic mandibles. J Prosthet Dent 1994;71:345-349

16. Blahout RM, Hienz S, Solar P, et al: Quantification of bone resorption in the interforaminal region of the atrophic mandible. Int J Oral Maxillofac Implants 2007;22:609-615

17. Cordioli G, Majzoub Z: Heat generation during implant site preparation: an in vitro study. Int J Oral Maxillofac Implants 1997; 12:186-193

18. Albrektsson T, Wennerberg A: Oral implant surfaces: Part 1-review focusing on topographic and chemical properties of different surfaces and in vivo responses to them. Int $\mathrm{J}$ Prosthodont 2004;17:536-543 
19. Scarano A, Carinci F, Quaranta A, et al: Effects of bur wear during implant site preparation: an in vitro study. Int J Immunopathol Pharmacol 2007;20:23-26

20. Meijer HJ, Starmans FJ, Steen WH, et al: A three-dimensional finite element study on two versus four implants in an edentulous mandible. Int J Prosthodont 1994;7:271-279

21. Papavasiliou G, Kamposiora P, Bayne SC, et al: Three-dimensional finite element analysis of stress-distribution around single tooth implants as a function of bony support, prosthesis type, and loading during function. J Prosthet Dent 1996;76:633-640

22. Hedia HS: Stress and strain distribution behavior in the bone due to the effect of cancellous bone, dental implant material and the bone height. Biomed Mater Eng 2002;12:111-119

23. Chang MC, Ko CC, Liu CC, et al: Elasticity of alveolar bone near dental implant-bone interfaces after one month's healing. J Biomech 2003;36:1209-1214

24. Korioth TW, Hannam AG: Deformation of the human mandible during simulated tooth clenching. J Dent Res 1994;73:56-66

25. Ivanoff CJ, Sennerby L, Lekholm U: Influence of mono- and bicortical anchorage on the integration of titanium implants. A study in the rabbit tibia. Int J Oral Maxillofac Surg 1996;25: 229-235

26. Jeong CM, Caputo AA, Wylie RS, et al: Bicortically stabilized implant load transfer. Int J Oral Maxillofac Implants 2003;18:59-65

27. Pierrisnard L, Renouard F, Renault P, et al: Influence of implant length and bicortical anchorage on implant stress distribution. Clin Implant Dent Relat Res 2003;5:254-262

28. Kitagawa T, Tanimoto Y, Nemoto K, et al: Influence of cortical bone quality on stress distribution in bone around dental implant. Dent Mater J 2005;24:219-224

29. Miyamoto I, Tsuboi Y, Wada E, et al: Influence of cortical bone thickness and implant length on implant stability at the time of surgery-clinical, prospective, biomechanical, and imaging study. Bone 2005;37:776-780

30. Himmlova L, Dostalova T, Kacovsky A, et al: Influence of implant length and diameter on stress distribution: a finite element analysis. J Prosthet Dent 2004;91:20-25

31. Van Der Zel JM: Implant planning and placement using optical scanning and cone beam CT technology. J Prosthodont 2008; 17:476-481 